American Journal of Neuroscience 2 (2): 59-64, 2011

ISSN 1948-9900

(C) 2011 Science Publications

\title{
Motor Imagery and Stroke Neurorehabilitation: An Overview of Basic Concepts and Therapeutic Effects
}

\author{
${ }^{1}$ Amanda Lopes Abbas, \\ ${ }^{1}$ Marina Fortuna Lucas, ${ }^{1}$ Silmar Teixeira, ${ }^{2,3,7}$ Flavia Paes, \\ ${ }^{1,4}$ Bruna Velasques, ${ }^{1,4,5}$ Pedro Ribeiro, ${ }^{2,7}$ Antonio E. Nardi and ${ }^{2,6,7}$ Sergio Machado \\ ${ }^{1}$ Institute of Psychiatry, Brain Mapping and Sensory Motor Integration, \\ Federal University of Rio de Janeiro (IPUB/UFRJ), Brazil \\ ${ }^{2}$ Institute of Psychiatry, Panic and Respiration Laboratory, \\ Federal University of Rio de Janeiro (IPUB/UFRJ), Brazil \\ ${ }^{3}$ Faculty of Psychology, \\ Brazilian Institute of Medicine and Rehabilitation, Rio de Janeiro, Brazil \\ ${ }^{4}$ Institute of Applied Neuroscience (IAN), Rio de Janeiro, Brazil \\ ${ }^{5}$ School of Physical Education, Bioscience Department (EEFD/UFRJ), Brazil \\ ${ }^{6}$ Quiropraxia Program, Central University, Santigo, Chile \\ ${ }^{7}$ National Institute for Translational Medicine (INCT-TM), Brazil
}

\begin{abstract}
Problem statement: Motor Imagery (MI), which corresponds to an active process during which the representation of a specific action is internally reproduced into working memory without any motor output. It represents the result of conscious access to the content of a movement intention, which is usually performed unconsciously during movement preparation. Approach: This review study aims to provide information on the current research and main findings related to the potential therapeutic effects of motor imagery on stroke neurorehabilitation. Results: Several studies demonstrate that conscious motor imagery and unconscious motor preparation share common mechanisms and are functionally equivalent, improving recovery of motor skills in stroke patients. Conclusion: In conclusion, motor imagery, proved very useful and effective, with significant results in improvement of motor deficits in post stroke patients. Thus, it is recommended that further studies must be conducted to determine specific parameters such as number and weekly frequency, duration (minutes per session), type (visual or kinesthetic) and the appropriate moment to apply mental practice (phases recovery of pathology), in order to create specific protocols for each treatment phase.
\end{abstract}

Key words: Brain, motor deficits, motor imagery, neurorehabilitation, stroke

\section{INTRODUCTION}

Stroke is defined as a sudden, focal neurological deficit due to a cerebrovascular abnormality. Stroke can cause substantial motor dysfunction that compromises ability to perform valued activities. It is considered one of the major causes of functional limitation in the world (Rabadi, 2010; Jittiwat et al., 2009; Hamed et al., 2007; Butler and Page, 2006; Cicerone et al., 2005). Over the years, the number of people affected by stroke has increased substantially due to the aging of world population. The specific deficits seen after stroke depend on the area of the brain affected. Hemiparesis, hemiplegia, aphasia, dysarthria, dysphagia, neglect, pain, cognitive deficits, sensory loss and depression are common and can be extremely limiting (Stevens and Stoykoy, 2003).

Although several methods of rehabilitation are currently used to facilitate movement in the affected extremity and teach compensatory techniques to perform Activities of Daily Living (ADLs), many patients remain with sensorimotor deficits and unable to perform ADLs. Within this context, motor imagery started to be used in the 80 's, however not so widely, as a potential treatment approach in post stroke patients (Deutsch et al., 2005). Studies have shown that motor imagery belongs to the same category of processes that would involve programming, planning and preparation

Corresponding Author: Sergio Machado, Institute of Psychiatry, Panic and Respiration Laboratory, Federal University of Rio de Janeiro (IPUB/UFRJ), Av. Venceslau Brás 71, Fundos. Botafogo, CEP 22290-140, Brazil 
for motor tasks, but without them being executed (Dechent et al., 2005).

Motor Imagery (MI) corresponds to an active process during which the representation of a specific action is internally reproduced into working memory without any motor output (Deepa et al., 2011; Lotze and Cohen, 2006; Dietrich, 2008). It represents the result of conscious access to the content of a movement intention, which is usually performed unconsciously during movement preparation (Gentili et al., 2006; Jackson et al., 2001; Dietrich, 2008). It is used as a cognitive rehearsal of physical movements and has emerged as a promising technique to improve motor skill performance, both in healthy people and people with motor deficits (Jackson et al., 2001; Braun et al., 2006). Several studies demonstrate that conscious motor imagery and unconscious motor preparation share common mechanisms and are functionally equivalent, improving recovery of motor skills in stroke patients (Milton et al., 2008). MI plays an important role for re-organization of neural network, strictly related to the motor strategy planned by the subject (Jackson et al., 2001). Moreover, it is well known that individual corticospinal cells innervate the motor neurons of multiple hand muscles. Thus, it is likely that a function of MI for motor learning is sensory-motor gating for the role of peripheral sensory input on the plasticity of motor cortical re-organization (Decety and Grezes 2006; Mulder, 2007; Page et al., 2007). Within this context, this review study aims to provide information on the current main research and findings related to the potential therapeutic effects of motor imagery on Stroke Neurorehabilitation.

Motor imagery: Specifically, $\mathrm{MI}$ is the mental representation of a movement without any motor output (Mulder, 2007). It is a complex cognitive operation that is self-generated by sensory and perceptual processes, allowing the reactivation of certain motor actions in working memory (Dickstein and Deutsch, 2007). It consists of a training method by which the internal reproduction of a given motor act (i.e., mental simulation) is repeated extensively with the intention of promoting learning or improving a motor skill (Stevens and Stoykoy, 2003).

It is a dynamic state during which the representation of a specific motor action is internally activated without any motor output (Braun et al., 2006; Milton et al., 2008; Munzert et al., 2009). The motor images require conscious activation of brain regions that are also involved in the preparation and performance of the movement, accompanied by a voluntary inhibition of the real movement (Lotze and
Cohen 2006). Studies have shown that there is similarity in the physiological and psychophysical functions between executed and imagined movements, suggesting that they are based on the same process. Based on this rationale, many investigators have proposed using $\mathrm{MI}$ in neurorehabilitation as a costefficient means to promote motor recovery after damage to the Central Nervous System (CNS) (Zimmermann-Schlatter et al., 2008).

A considerable number of electromyographic studies have reported that the patterns of muscular activation during $\mathrm{MI}$ are strikingly similar to those exhibited during physical execution of the same movements (Page et al., 2005; Mulder, 2007). In addition, some studies showed that images produce an identical, minute innervation in the targeted musculature as if the same movement were being physically performed. Interesting, muscles are proportional activated according to degree of imagined effort, e.g., a more vigorous task during MI elicits greater electromyographic amplitude than a less vigorous task (Grush, 2004).

With the advent of brain mapping techniques for instance functional Magnetic Resonance Imaging (fMRI) and Electroencephalography (EEG) allowed for first time a more precise anatomic localization of the cerebral structures implicated in performing imagined and executed movements mainly of the upper limb (Mulder, 2007). Altogether, these findings indicate that the Supplementary Motor Area (SMA), cerebellum, as well as the premotor, cingulate, superior and inferior parietal and primary motor cortex (M1) are often found to be involved in both the execution and the imagination of upper limb movements (Jackson et al., 2001; Dechent et al., 2004).

These findings support the idea that the structures or systems mediating the simulation of motor activity and the motor execution are alike. The similarities between MI and executed movements can explain the motor performance improvements exhibit after MI (Jackson et al., 2011; Page et al., 2007) and could indicate that the MI as promising technique to improve motor skills performance. However, further investigations are still necessary to determine the potential use of MI in neurological rehabilitation particularly of people with stroke.

Types of motor imagery: MI can be classified as kinesthetic and visual (Verbunt et al., 2008). Kinesthetic MI is performed in first person, which performer approximates a real life situation in such a way that the person actually experiences the sensory sensations), providing the feeling of movements as if they were really 
performed. Indeed, it requires an approximation of the real life phenomenology such that the person actually imagines being inside his/her body and experiencing those sensations that might be expected in the actual situation. On the other hand, visual MI is performed in third person (related to the scenes outside the person) providing the mental visualization of the movement performance as from a distance, actually the subject views himself from the perspective of an external observer (Guillot and Collet, 2005).

The use of kinesthetic or visual MI types seems to be influenced by two relevant factors associated with rehabilitation process; the nature of the task and the stage of learning (Vries and Mulder, 2007). For instance, to learn a new motor task, visual MI is more suitable for tasks that emphasized form, whereas kinesthetic MI is better for those tasks that emphasized timing or coordination of hands' movement (Byrne et al., 2007). Both perspectives were associated with the activation of common neural networks in the SMA, the precentral gyrus and the precuneus. First perspective taking, however, was associated with the increased activity in the left inferior parietal lobule and the left somatosensory cortex, whereas the third person perspective activated the right inferior parietal lobule, the posterior cingulate and the fronto-polar cortex (Vries and Mulder, 2007; Mulder, 2007).

In addition, several factors must be taken into consideration in the decision about which MI category (i.e., visual or kinesthetic) to use in therapy. The first factor is that imagery of human movement is a cognitive operation that depends on the dynamic relationship among the individual, the movement and the environment (Byrne et al., 2007). In addition, imagery perspectives should be determined by the nature of the task, the environment and individual characteristics. Considering that the separation into visual and kinesthetic MI is highly artificial, the application of both visual and kinesthetic imagery appears feasible and appropriate for most individuals (Guillot and Collet, 2005).

Motor Imagery ability: In order to optimize the benefits of the MI practice, the ability to use the MI is a relevant consideration. Several recommendations and reservations about the screening of patients for MI practice have been discussed in the literature (Dickstein and Deutsch, 2007). The parietal lobe is well-known as the main brain area responsible for the generation of mental representations of movement. The ability to generate imagined movements is necessary for MI to be most effective. Some studies indicate that lesions in parietal lobes can impair MI, implying that patients with neurological diseases may not benefit from it (Jackson et al., 2001). For instance, patients with parietal and left lateral prefrontal lesions are not able to imagine a movement (Lotze and Cohen, 2006). In addition, damage to the basal ganglia also influences the ability to perform MI (Dickstein and Deutsch, 2007). Another factor that supposedly influence on the ability to perform MI is the age. Gabbard et al. (2011) instructed young and old participants to estimate, using MI, whether randomly presented targets in peripersonal (i.e., within actual reach) and extrapersonal (i.e., beyond reach) space were within or out of reach of their dominant limb. The findings showed that old subjects are less accurate than the young subjects making more errors in extrapersonal space during performance of MI. Thus, it seems there is a decline in the ability to perform MI in aging.

\section{Factors that influence on motor imagery practice:}

Task familiriaty: Task familiarity is a prerequisite for the successful use of MI (Verbunt et al., 2008). Mulder (2007) observed that after MI individuals who had previously mastered a new motor task improved substantially compared to individuals with no previous practice. In a similar study, Mutsaarts et al. (2006) showed that individuals with hemiparetic cerebral palsy and deficits in the ability of MI compared to control individuals were unable to plan new tasks. However, the study by Aleman et al. (2001) explored the ability of individuals with congenital total blindness compared to healthy individuals through performance of visual MI tasks. With regard to the task familiarity, the congenitally blind subjects are able to perform visual MI, though made more errors. Imbiriba et al. (2006) made similar conclusions for blind individuals. Thus, clearly there is conflicting information regarding the role of familiarity in the successfully using MI due to several definitions of terms such as "motor tasks" and "novelty". Therefore, generalizations from a study related to other populations or conditions should be analyzed with caution. Nevertheless, the notion that the effectiveness of MI is related to task familiarity and to familiar tasks being associated with better performance than unfamiliar tasks should be considered when selecting patients and planning an intervention (Bohan et al., 1999).

Working memory: Working memory is a complex process that includes the on-line storage and active manipulation of information, which can be categorized as visual, verbal, or kinesthetic. It is generally conceived as a multicomponent system, which relies on a complex network of brain areas (Malouin et al., 
2003). Lotze and Cohen (2006) described motor imagery as a dynamic process during which the representation of a specific action is internally reactivated, supporting that mental rehearsal requires that individuals maintain and manipulate visual and kinesthetic information in their working memory. Impairment in working memory, therefore, may impair the ability to participate successfully in MI (Vries and Mulder, 2006). Malouin et al. (2003) observed that the improvement in task performance of standing up/ sitting down after intervention of physical therapy combined with MI was reinforced in a group with intact working memory compared to a group with deficits in working memory. The strongest relationship was found in the visual-spatial domain, followed by verbal and kinesthetic domains. Despite, it is noteworthy that the practice of MI combined with physical practice may improve the performance of an anticipatory motor task more than the physical practice itself in individuals with a high probability of deficits in working memory (Byrne et al., 2007).

Effects of motor imagery on stroke neurorehabilitation: Conventional rehabilitative strategies have been typically focused on compensation with the unaffected limb and/or non-functional exercises involving the affected limb. However, emphasis has been given on Repetitive Task Practice (RTP) to produce motor changes, e.g., MI, even years after stroke. Despite the explosion of these types of techniques, e.g. Constraint Induced Movement (CIT), MI is still considered the most cost-effective, easily implemented and non-invasive technique in which the motor skills of patients are cognitively rehearsed in absence of physical movements (ZimmermannSchlatter et al., 2008). Thus, MI can be considered as a use-dependent brain reorganization process, where new cortical areas would be recruited for assisting motor restoration of the affected arm (Munzert et al., 2009).

The use of MI to improve motor function after stroke has gained much attention over the last decade (Dickstein and Deutsch, 2007). Braun et al. (2006) showed that there is evidence that MI, as an additional therapy, has effects on motor recovery after stroke. Although the ability to engage in motor imagery is required for $\mathrm{MI}$, however, in most of the reported studies MI ability was not assessed (Jackson et al., 2001; Malouin et al., 2003; Sharma et al., 2006; Braun et al., 2006).

Page et al. (2001) in a case repots investigated the effects of MI combined with physical therapy in 2 subacute stroke patients. The patient A received a protocol of stroke information plus physical therapy compared to patient B that received MI plus physical therapy. The physical therapy was performed 3 days per week for 6 weeks and MI and stroke information 2 days per week for 6 weeks. Again, after the period of treatment, patient $\mathrm{B}$ exhibited a significant improvement in fine motor skills compared to patient A. Liu et al. (2004) in a randomized controlled study used a MI program based on the functional retraining program used as a control intervention, thus limiting inclusion to stroke patients with a degree of movement. After 15 sessions of training, significant outcomes were achieved, indicating an improvement in the functional status of patients. Another study, conducted by Page et al. (2005), investigated the effects of MI combined with physical therapy in 6 chronic stroke patients. Patients were trained for 30 minutes 2 days per week for 6 weeks, the experimental group received MI plus physical therapy while the control group received relaxation exercises plus physical therapy. The findings indicated that the experimental group improved the function of the impaired arm when compared to the control group, indicating that MI can be considered a mechanism of reinforce sensorimotor integration process, even in chronic patients. Recently, Page et al. (2007) provides the first randomized controlled study, appropriately powered support to the hypothesis that substantive motor changes may be produced through a regimen including MI. Patients were trained for 30 minutes 2 days per week for 6 weeks in both groups. The authors demonstrated that the combination of MI and physical therapy in chronic patients is effective for upper limb motor restoration in chronic stroke patients.

It seems MI demands a conscious engagement of certain brain regions often activated unconsciously during motor preparation. However, MI is not dependent on motor execution skills, but it is quite a lot of dependent on processing of central mechanisms (Jeannerod, 1995). With this in mind, it may be possible that the frequent use of MI facilitate the central motor commands organization. Based on the "neural networks" theory, which underlines that they are previously established for certain motor acts, studies have been reported that those neural networks involved in motor gesture execution are rehearsal during MI (Kosslyn et al., 1995). Thus, the improvement in performance of the executed motor gesture occurs by coordination of motor patterns responsible for its development. It is based on the theory that "neural networks" remains intact despite the physical damages, which suggest that post stroke patients could benefit of MI use activating the partially damaged "neural networks" (Jeannerod and Decety, 1995). Those findings are in agree with previous studies of MI, despite the lack of neuroimaging data to reinforce this 
rationale (Jeannerod, 2001), however, a few studies showed changes in cortical functions when specific protocol of motor tasks were used, including MI (Lotze and Cohen, 2006). Such theory is attributed to a mechanism cerebral reorganization, where new areas are recruited to assist the movements of the affected arm (Jeannerod, 2001; Lotze and Cohen, 2006).

Thus, MI prior to motor execution would represent an additional or complementary technique to motor execution, but do not replace it. Since the patients' ability to perform the MI is evaluated, the focus can then be directed on the severity of the injury and the moment when the MI should be introduced to the treatment. When the neurological condition does not allow patients to perform movements, MI is needed in order to keep the neural networks active and also to promote cortical reorganization, so that the motor preparation facilitates future executions of specific movements during the rehabilitation program.

\section{CONCLUSION}

Motor imagery proved very useful and effective, with significant results in improvement of motor deficits in post stroke patients. However, there is no consensus about a treatment protocol using MI for neurorehabilitation of stroke patients. Thus, it is recommended that further studies must be conducted to determine specific parameters such as number and weekly frequency, duration (minutes per session), type (visual or kinesthetic) and the appropriate moment to apply mental practice (phases recovery of pathology), in order to create specific protocols for each treatment phase. Moreover, new studies must be performed using neuroimaging techniques in order to obtain more information about the patterns of activation and reorganization of the brain.

\section{REFERENCES}

Aleman, A., L.V. Lee, M.H. Mantione, I.G. Verkoijen and E.H. De Haan, 2001. Visual imagery without visual experience: Evidence from congenitally totally blind people. Neuroreport, 12: 2601-4. PMID: 11496156

Bohan, M., J.A. Pharmer and A.F. Stokes, 1999. When does imagery practice enhance performance on a motor task? Percept Mot Skills, 88: 651-658. PMID: 10483658

Braun, S.M., A.J. Beurskens, P.J. Borm, T. Schack and D.T. Wade, 2006. The effects of mental practice in stroke rehabilitaton: A systematic review. Arch. Phys. Med. Rehabil., 87: 842-852. PMID: 16731221
Butler, A.J. and S.J. Page, 2006. Mental practice with motor imagery: Evidence for motor recovery and cortical reorganization after stroke. Arch. Phys. Med. Rehabil., 87: S2-S11. PMID: 17140874

Byrne, P., S. Becker and N. Burgess, 2007. Remembering the past and imagining the future: a neural model of spatial memory and imagery. Psychol. Rev., 114: 340-375. PMID: 17500630

Cicerone, K.D., C. Dahlberg, J.F. Malec, D.M. Langenbahn and T. Felicetti et al., 2005. Evidencebased cognitive rehabilitation: Updated review of the literature from 1998 through 2002. Arch. Phys. Med. Rehabil., 86: 1681-1692. PMID: 16084827

Vries, S.D. and T. Mulder, 2007. Motor imagery and stroke rehabilitation: A critical discussion. J. Rehabil. Med., 39: 5-13. PMID: 17225031

Decety, J. and J. Grezes, 2006. The power of simulation: Imagining one's own and other's behavior. Brain Res., 1079: 4-14. PMID: 16460715

Dechent, P., K.D. Merboldt and J. Frahm, 2004. Is the human primary motor cortex involved in motor imagery? Brain Res. Cogn. Brain Res., 19: 138144. PMID: 15019710

Deepa, V.B., P. Thangaraj and S. Chitra, 2011. Classifying single trail electroencephalogram using gaussian smoothened fast hartley transform for brain computer interface during motor imagery. J. Comput. Sci., 7: 757-761. DOI: 10.3844/jcssp.2011.757.761

Deutsch, J.E., S. Fischer, W. Liu, A. Kalnin and K. Mosier, 2005. Representation of imagined and executed sequential finger movements of adults post stroke and healthy controls. J. Neurol. Phys. Ther., 29: 205-205. DOI: 10.1097/01.NPT.0000282371.70655.d4

Dickstein, R. and J.E. Deutsch, 2007. Motor Imagery in Physical Therapist Practice. Phys. Ther., 87: 942953. PMID: 17472948

Dietrich, A., 2008. Imaging the imagination: The trouble with motor imagery. Methods, 45: 319-324. PMID: 18539161

Gabbard, C., P. Caçola and A. Cordova, 2011. Is there an advanced aging effect on the ability to mentally represent action? Arch. Gerontol. Geriatr., 53: 206209. PMID: 21035202

Gentili, R., C. Papaxanthis and T. Pozzo, 2006. Improvement and generalization of arm motor performance through motor imagery practice. Neuroscience, 137: 761-772. PMID: 16338093

Grush, R., 2004. The emulation theory of representation: Motor control, imagery and perception. Behav. Brain Sci., 27: 377-396. PMID: 15642689 
Guillot, A. and C. Collet, 2005. Duration of mentally simulated movement: A review. J. Mot. Behav., 37: 10-20. PMID: 15642689

Hamed, M.A., Z.X. Hong, X. Bo, S. Zhi and T.Q. Yun, 2007. Acute and subacute phase of cerebral infarction after treatment with acupuncture and medical therapy. Am. J. Infec. Dis., 3: 7-10. DOI: 10.3844/ajidsp.2007.7.10

Imbiriba, LA., E.C. Rodrigues, J. Magalhaes and C.D. Vargas, 2006. Motor imagery in blind subjects: The influence of the previous visual experience. Neurosci. Lett., 400: 181-185. PMID: 16530330

Jackson, P.L., M.F. Lafleur, F. Malouin, C. Richards and J. Doyon, 2001. Potential role of mental practice using motor imagery in neurologic rehabilitation. Arch. Phys. Med. Rehabil., 82: 1133-1141. PMID: 11494195

Jeannerod, M. and J. Decety, 1995. Mental motor imagery: A window into the representational stages of action. Curr. Opin. Neurobiol., 5: 727-732. PMID: 8805419

Jeannerod, M., 1995. Mental imagery in the motor context. Neuropsychologia, 33: 1419-1132. PMID: 8584178

Jeannerod, M., 2001. Neural simulation of action: A unifying mechanism for motor cognition. NeuroImage, 14: S103-S109. PMID: 11373140

Jittiwat, J., J. Wattanathorn, T.T.S. Muchimapura and C. Bunchonglikitkul, 2009. Porcine brain extract attenuates memory impairments induced by focal cerebral ischemia. Am. J. Applied Sci., 6: 16621668. DOI: 10.3844/ajassp.2009.1662.1668

Kosslyn, S.M., M. Behrmann and M. Jeannerod, 1995. The cognitive neuroscience of mental imagery. Neuropsychologia, 33: 1335-1344. PMID: 8584172

Liu, K.P., C.C. Chan, T.M. Lee and C.W. Hui-Chan, 2004. Mental imagery for promoting relearning for people after stroke: A randomized controlled trial. Arch. Phys. Med. Rehabil., 85: 1403-1408. PMID: 15375808

Lotze, M. and L.G. Cohen, 2006. Volition and imagery in neurorehabilitation. Cogn. Behav. Neurol., 19: 135-140. PMID: 16957491

Malouin, F., C.L., Richards, P.L. Jackson, F. Dumas and J. Doyon, 2003. Brain activations during motor imagery of locomotor-related tasks: A PET study. Hum. Brain Mapp., 19: 47-62. PMID: 12731103

Milton, J., S.L. Small and A. Solodkin, 2008. Imaging motor imagery: Methodological issues related to expertise. Methods, 45: 336-341. PMID: 18762138
Mulder, T., 2007. Motor imagery and action observation: Cognitive tools for rehabilitation. J Neural Transm., 114: 1265-1278. DOI: 10.1007/s00702-007-0763-z

Munzert, J., B. Lorey and K. Zentgraf, 2009. Cognitive motor processes: The role of motor imagery in the study of motor representations. Brain Res. Rev., 60: 306-326. PMID: 19167426

Mutsaarts, M., B. Steenbergen and H. Bekkering, 2006. Anticipatory planning deficits and task context effects in hemiparetic cerebral palsy. Exp. Brain. Res., 172: 151-162. PMID: 16538378

Page, S.J., P. Levine, A.S. Sisto and M.V. Johnston, 2001. Mental practice combined with physical practice for upper-limb motor deficit in subacute stroke. Phys. Ther., 81: 1455-1462. PMID: 11509075

Page, S.J., P. Levine and A.C. Leornard, 2005. Effects of mental practice on affected limb use and function in chronic stroke. Arch. Phys. Med. Rehabil., 86: 399-402. PMID: 15759218

Page, S.J., P. Levine and A. Leonard, 2007. Mental practice in chronic stroke: Results of a randomized, placebo-controlled trial. Stroke, 38: 1293-1297. PMID: 17332444

Rabadi, M.H., 2010. Randomized Clinical stroke trials in 2008. Am. Med. J., 1: 8-22. DOI: 10.3844/amjsp.2010.8.22

Sharma, N., V.M. Pomeroy and J.C. Baron, 2006. Motor imagery: A backdoor to the motor system after stroke? Stroke, 37: 1941-1952. PMID: 16741183

Stevens, J.A. and M.E. Stoykoy, 2003. Using motor imagery in the rehabilitation of hemiparesis. Arch. Phys. Med. Rehabil., 84: 1090-1092. PMID: 12881842

Verbunt, J.A., H.A. Seelen, F.P. Ramos, B.H. Michielsen and W.L. Wetzelaer et al., 2008. Study protocol-mental practice-based rehabilitation training to improve arm function and daily activity performance in stroke patients: A randomized clinical trial. BMC Neurol., 8: 7-7. DOI:10.1186/1471-2377-8-7 PMID: 18405377

Zimmermann-Schlatter, A., C. Schuster, M.A. Puhan, E. Siekierka and J. Steures, 2008. Efficacy of motor imagery in post-stroke rehabilitation: A systematic review. J. Neuroeng. Rehab., 5: 8-8. DOI: 10.1186/1743-0003-5-8 PMID: 18341687 